\title{
COVID-19 and Antiretroviral Therapies: South Africa's Charge Towards 90-90-90 in the Midst of a Second Pandemic
}

\author{
Andrea S. Mendelsohn ${ }^{1} \cdot$ Tiarney Ritchwood ${ }^{2}$
}

Published online: 30 April 2020

(c) Springer Science+Business Media, LLC, part of Springer Nature 2020

The COVID-19 pandemic has spurred panic in South Africa, a country with the highest number of HIV patients in the world and a persistent TB epidemic. South Africa had its first COVID-19 case on March 5, 2020. Fearful that a significant segment of the population was particularly vulnerable-7.7 million South Africans have HIV and even more live in crowded conditions - the president declared a national state of disaster on March 15th when the caseload rose to 51 [1]. The country initially implemented a 3-week lockdown to contain COVID-19's spread, which was later extended to 5 weeks. In preparation for a future swell of COVID-19 patients, the Western Cape Department of Health (WC DoH) implemented a plan to "de-escalate" healthcare services to reduce the spread of infection and increase capacity to accommodate COVID-19 patients [2]. All non-urgent elective surgeries and outpatient appointments were postponed. Stable chronic patients were given 2-months supplies of medication with up to 6 months of refills. Only emergency life-saving care, contraception, antenatal care, and immunizations have continued as normal.

Telemedicine is not a viable alternative in the South African public health system. Consequently, all patients are screened at the hospital gate for acute respiratory symptoms before entering. Those with possible COVID-19 symptoms are given surgical masks and isolated for clinical assessment, treatment and testing. Inside the hospital, social distancing is adhered to as strictly as possible. In one clinic, rather than sitting in a crowded waiting area, patients are asked to wait $1.5 \mathrm{~m}$ apart from each other outside and brought into the building for their visit or pharmacy collection 5 patients at a time. As much as possible, chronic medications

Andrea S. Mendelsohn

andrea.mendelsohn@westerncape.gov.za

1 Western Cape Department of Health, Cape Town, South Africa

2 Department of Family Medicine and Community Health, Duke University, Durham, NC, USA are packaged and delivered by community health workers to community-based locations.

The same de-escalation plan makes provision for the distribution of anti-retroviral medication (ARVs) to the 5 million South Africans currently on ARVs [1]. While there is no evidence that people living with HIV (PLHIV) who are virally suppressed on ARVs are at increased risk of severe COVID-19 disease, there is genuine concern that PLHIV with advanced immunocompromise or unsuppressed HIV may suffer worse COVID-19 outcomes [3]. Therefore, there is extra pressure in South Africa to decrease the exposure of PLHIV to COVID-19 infection and rapidly increase viral suppression rates.

Further complicating the pandemic is South Africa's rollout of a new first-line ARV, a fixed-dose combination pill containing tenofovir disoproxil fumarate, lamivudine, and dolutegravir (TLD). South Africa has changed to a first line dolutegravir-based regimen because of its higher barrier to resistance and reduction in associated side effects compared to efavirenz. In 2019 the change to TLD was delayed over concerns of increased neural tube defects when women conceive on TLD. However, reassured by improved Botswana data and persuaded that ARV programs must respect a woman's right to make her own healthcare decisions, the South African National Department of Health launched TLD on December 1, 2019 [4-6]. Providers began prescribing TLD nationwide in January 2020.

Prior to the COVID-19 outbreak, the plan in South Africa was to phase out the old first-line fixed dose combination pill of tenofovir disoproxil fumarate, emtricitabine, and efavirenz (TEE) slowly over 1-2 years. Since TEE stocks in the Western Cape are limited and TLD is plentiful, it is essential to roll-out TLD as planned [7]. What's more, TLD is a more effective ARV $[8,9]$. During a highly infectious respiratory pandemic, PLHIV should be on the best ARV regimen available to minimize their risk of virologic failure, immunocompromise, and severe COVID-19 infection. 
To facilitate the rollout of TLD with de-escalated HIV services, the WC DoH and the Southern African HIV Clinicians Society (SAHCS) issued specific recommendations [7, 10]. The SAHCS strongly advocated that 6-month supplies of ARVs be issued to stable patients, reducing the risk of COVID-19 exposure inherent in seeking in-person treatment at healthcare facilities as well as to minimize patient flow in a clinic [10]. Ideally, to change from TEE to TLD a patient should have a $\mathrm{VL}<50$ copies/mL within 6 months, as stipulated by national guidelines. However, the SAHCS and WC DoH advocated switching a patient to TLD, even without a recent VL, if the patient has been on ARVs for $>1$ year, the past 2 VLs were $<50$ copies $/ \mathrm{mL}$, and they have regularly collected ARVs over the past year [7, 10]. Patients fulfilling these criteria are very likely to be suppressed and can be switched to TLD without delay [10]. Additionally, the WC DoH urged same day initiation of ARVs when not medically contraindicated, ARV initiation for all co-infected TB patients 2 weeks after starting TB treatment, and urgently changing all patients failing $1^{\text {st }}$ line TEE to a $2^{\text {nd }}$ line ARV regimen, preferably with dolutegravir [7]. Patients unsuppressed on ARVs will need additional clinical contact and adherence support. The SAHCS and WC DoH recommendations seek to fast-track PLHIV onto a robust ARV regimen during the pandemic to maximize their possibility of viral suppression and minimize their exposure to the healthcare system and, potentially, COVID-19.

The uncertainty is what impact this de-escalated HIV program will have on HIV outcomes in South Africa. Unsuppressed patients in need of additional adherence support are likely to suffer the most without the help of auxiliary services such as social work, treatment support groups, addiction rehabilitation, and psychotherapy. Likewise, many of South Africa's lay HIV-counselors have been re-deployed to community COVID-19 screening. Although HIV testing is ongoing, anecdotally testing numbers and new diagnoses of HIV are lower since the WC DoH began discouraging nonessential healthcare visits. HIV self-testing at home or HIV testing in coordination with community COVID-19 screens could potentially fill that gap.

It is well documented that differentiated models of care, such as South Africa's adherence clubs, for stable ARV patients have improved long-term virologic suppression and retention in care in comparison to usual care [11-13]. PLHIV are more likely to continue treatment if it is convenient and they can keep working without sitting all day in un-friendly, stigmatizing clinics to collect medication. In fact, the WHO recommends that clinically stable patients receive ARV refills every 3-6 months to decrease the burden of care [14]. Forced by the pandemic, the WC DoH has advised clinicians to give stable patients up to 6 months of refills of a highly efficacious ARV_-TLD—with minimal clinic contact. We have effectively turned most of the HIV service into one giant alternative model of care. It is possible that without the burden of frequent visits, there might be improved retention in care during the pandemic because patients are given the medication and empowered to manage their own health. The roll-out of TLD will confound outcomes, because it will be impossible to tease out if viral suppression rates are due to the better ARV, less burdensome healthcare system, or both.

Crisis is often the catalyst for ingenuity. Depending on outcomes, we suspect that South Africa will ultimately return to a combination of the old and the new model of care. Stable ARV patients will be empowered to live their lives with 3-6 month supplies of medication and minimal clinic interaction. Those struggling with ARV adherence should be reabsorbed into multi-disciplinary teams to meet their medical, psychological, and social needs. Universal HIV testing should be implemented and TLD should be offered to all eligible PLHIV given its limited side effects and greater accessibility.

\section{References}

1. UNAIDS. South Africa Country Data. 2010. https://www.unaid s.org/en/regionscountries/countries/southafrica. Accessed $20 \mathrm{Apr}$ 2020 .

2. Engelbrecht, B, Cloete, K. Health Services Response to COVID-19. 2020. Western Cape Department of Health staff correspondence.

3. World Health Organization. Q\&A on COVID-19, HIV and antiretrovirals. https://www.who.int/news-room/q-a-detail/q-a-on-covid -19-hiv-and-antiretrovirals. 2020. Accessed 18 Apr 2020.

4. Zash R, Holmes L, Deseko M, et al. Neural-tube defects and antiretroviral treatment regimens in Botswana. NEJM. 2019;381:827-40.

5. World Health Organization. Consolidated guidelines on sexual and reproductive health and rights of women living with HIV. https ://www.who.int/reproductivehealth/publications/gender_rights/ Ex-Summ-srhr-women-hiv/en/. 2017. Accessed 17 Apr 2020.

6. UNITAID. Press Release: South Africa to introduce state-of-theart HIV treatment. https://unitaid.org/news-blog/south-africa-tointroduce-state-of-the-art-hiv-treatment/\#en. 2019. Accessed 18 Apr 2020.

7. Voget J, Mudaly V, Kruger J, Kariem S. Circular H37/2020. Management of Patients with HIV, TB and Non-Communicable Chronic Diseases During Outbreak of COVID-19; Multi-Month Dispensing of Chronic Medicines, including ART. Western Cape Department of Health; 2020.

8. Walmsley S, Antela A, Clumeck N, et al. Dolutegravir plus abacavir-lamivudine for the treatment of HIV-1 infection. N Engl J Med. 2013;369:1807-18.

9. Venter WDF, Moorhouse M, Sokhela S, et al. Dolutegravir plus two different prodrugs of tenofovir to treat HIV. N Engl J Med. 2019;381:803-15.

10. Provision of 6 months of antiretroviral treatment-position statement from the Southern African HIV Clinician Society. https ://sahivsoc.org/Files/SAHIVSoc_COVID_final2.pdf. 2020. Accessed 18 Apr 2020. 
11. Luque-Fernandez MA, Van Cutsem G, Goemaere E, et al. Effectiveness of patient adherence groups as a model of care for stable patients on antiretroviral therapy in Khayelitsha, Cape Town, South Africa. PLoS ONE. 2013;8(2):e56088.

12. Tsondai PR, Wilkinson LS, Grimsrud A, Mdlalo PT, Ullauri A, Boulle A. High rates of retention and viral suppression in the scale-up of antiretroviral therapy adherence clubs in Cape Town, South Africa. J Int AIDS Soc. 2017;20:51-7.

13. Wilkinson LS. ART adherence clubs: a long-term retention strategy for clinically stable patients on antiretroviral therapy. S Afr J HIV Med. 2013;14(2):48.
14. World Health Organization. Policy brief: consolidated guidelines on the use of antiretroviral drugs for treating and preventing HIV infections: what's new. https://apps.who.int/iris/handle/10665 /198064. 2015. Accessed 18 Apr 2020.

Publisher's Note Springer Nature remains neutral with regard to jurisdictional claims in published maps and institutional affiliations. 